\title{
Automated Calibration of Distributed Laser Range Finders Based on Object Tracking in Overlapping Sensing Regions
}

\author{
Takeshi Sasaki * Hideki Hashimoto* \\ * Institute of Industrial Science, The University of Tokyo, Tokyo, \\ Japan(e-mail:sasaki@ hlab.iis.u-tokyo.ac.jp, \\ hashimoto@iis.u-tokyo.ac.jp).
}

\begin{abstract}
In this paper, we address the automated calibration of the pose of distributed laser range finders in smart environments, which are spaces with multiple embedded and networked sensors and actuators. This method is based on object tracking in overlapping sensing regions: the positions of same tracked objects in each sensor's coordinate system are used to calculate relative position and orientation of the sensors. We focus on extension of this mobile-assisted approach in order to utilize general moving objects such as humans and not limited to mobile robots. In case that mobile robots are used as calibration objects, the model of the mobile robots can be used to determine which mobile robots are being tracked. However, if the general moving object is utilized, we have to judge whether two tracked objects in different sensors are same object or not. So estimation error is utilized for the decision on the corresponding object. Experimental results shows that this method can find the correct correspondence and achieve almost the same result as manual calibration case.
\end{abstract}

\section{INTRODUCTION}

In the recent years, the research field on smart environments, which are spaces with multiple embedded and networked sensors and actuators, has been expanding Cook and Das [2004]. The smart environments observe the space using distributed sensors, extract useful information from the obtained data and provide various services to users. Such an environment is also referred to as smart space, intelligent environment, etc., and under the concept of ubiquitous computing, many researchers have developed smart environments for providing informative services to the users (e.g. support during meeting Johanson et al. [2002], health care Nishida et al. [2000], support of the elderly Mynatt et al. [2004], information display using a pan-tilt projector Mori et al. [2004]). On the other hand, smart environments are also used for environment design for mobile robots to deal with complicated natural environments. Mobile robots inside smart environments can get necessary information from multiple distributed sensors and various functions such as localization, path planning and human-robot interaction are performed with the support of the space Mizoguchi et al. [1999], Koide et al. [2004], Sgorbissa and Zaccaria [2004].

However, one of the major problems in developing the multi-sensor system is calibration. Although calibration is needed for proper calculation from the local coordinate system to the world coordinate system, it takes a great deal of time and effort to calibrate many sensors. In order to solve this problem, we make use of moving objects in the space. Some researchers (e.g. Galstyan et al. [2004], Shenoy and Tan [2005]) focus on mobile-assisted node localization in wireless sensor network. In Rekleitis and Dudek [2005], mobile robots are utilized for calibration of a camera sensor network. The intrinsic and extrinsic camera parameters are calibrated based on grid patterns attached to a mobile robot. Howard et al. [2001] presented a generalized localization problem and its solution by relaxation on a mesh. As an application, distributed laser range finders are calibrated using a mobile robot. In Sasaki et al. [2007], we also described an automated calibration method of the pose of distributed laser range finders by using mobile robots. Mobile robots can cover wide areas of the environment so there is no need to place many landmarks in exactly known positions beforehand. However, the smart environments which are mainly focused on informative support may not have mobile robots. Furthermore, sensors may not be able to be calibrated immediately since calibration can be done only when mobile robots are found by sensors. So, in this paper, general moving objects not limited to mobile robots, such as humans, are utilized. The relative position and orientation of sensors are calculated based on the tracking result of moving objects in overlapping observable areas of different sensors.

The rest of the paper is organized as follows. Section 2 presents our intelligent environment, called Intelligent Space (iSpace) Lee and Hashimoto [2002]. The automated calibration method based on moving object tracking is explained in section 3. Experimental results are shown in section 4. Finally, conclusion and future work are given in section 5 .

\section{INTELLIGENT SPACE}

\subsection{Concept of Intelligent Space}

Fig. 1 shows the concept of Intelligent Space (iSpace), which is a space with multiple distributed and networked 


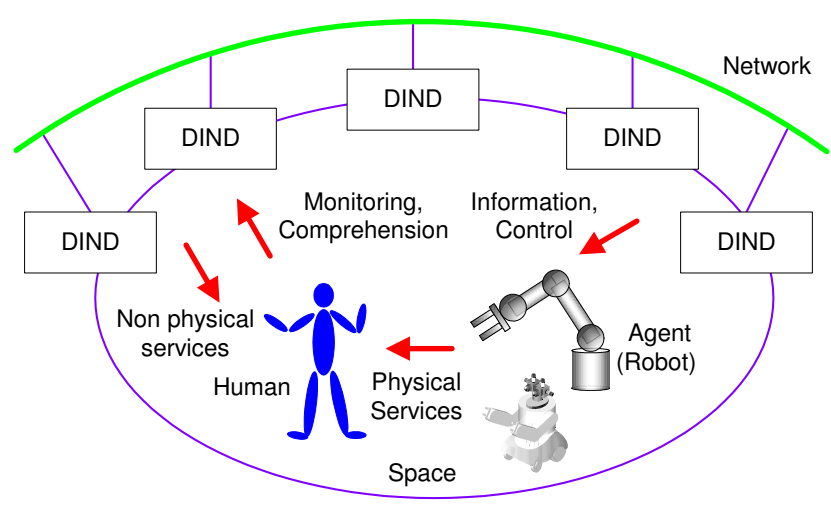

Fig. 1. Concept of Intelligent Space

sensors and actuators. In iSpace, not only sensor devices but also sensor nodes are distributed in the space because it is necessary to reduce the network load in the largescale network and it can be realized by processing the raw data in each sensor node before collecting information. We call the sensor node devices distributed in the space DINDs (Distributed Intelligent Network Device). A DIND consists of three basic components: sensors, processors and communication devices. The processors deal with the sensed data and extract useful information about objects (type of object, three dimensional position, etc.), users (identification, posture, activity, etc.) and the environment (geometrical shape, temperature, emergency, etc.). The network of DINDs can realize the observation and understanding of the events in the whole space. Based on the extracted and fused information, actuators such as displays or projectors embedded in the space provide informative services to users.

In iSpace, mobile robots are also used as actuators to provide physical services to the users and for them we use the name mobile agents. The mobile agent can utilize the intelligence of iSpace. By using distributed sensors and computers, the mobile agent can operate without restrictions due to the capability of on-board sensors and computers. Moreover, it can understand the request from people and offer appropriate services to them.

\subsection{Configuration of Intelligent Space}

Fig. 2 shows a picture of the implemented iSpace. iSpace is currently implemented in a laboratory environment which has an area of about 5 meters $\times 5$ meters. Three laser range finders, ten CCD cameras and a 3D ultrasonic positioning system are used as sensors of DIND.

The laser range finders are arranged in the space close to the ground (about $20 \mathrm{~cm}$ above the floor). The cameras are connected in pairs to computers with two video capture boards. As a result, each camera DIND can get the three dimensional position of objects by stereo vision. The 3D ultrasonic positioning system involves 96 ultrasonic receivers installed on the ceiling. This system can measure the three dimensional position of an ultrasonic transmitter to an accuracy of 20-80 millimeters using triangulation method. Moreover, a differential wheeled robot is used as mobile agent. For estimating the position and orientation of the robot, two ultrasonic transmitters are installed on

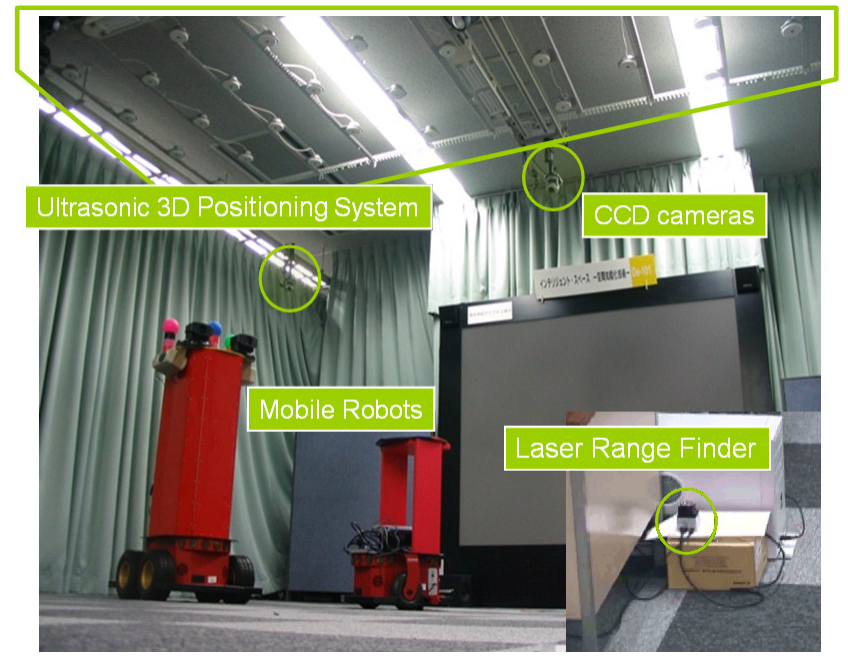

Fig. 2. Sensors and mobile agents

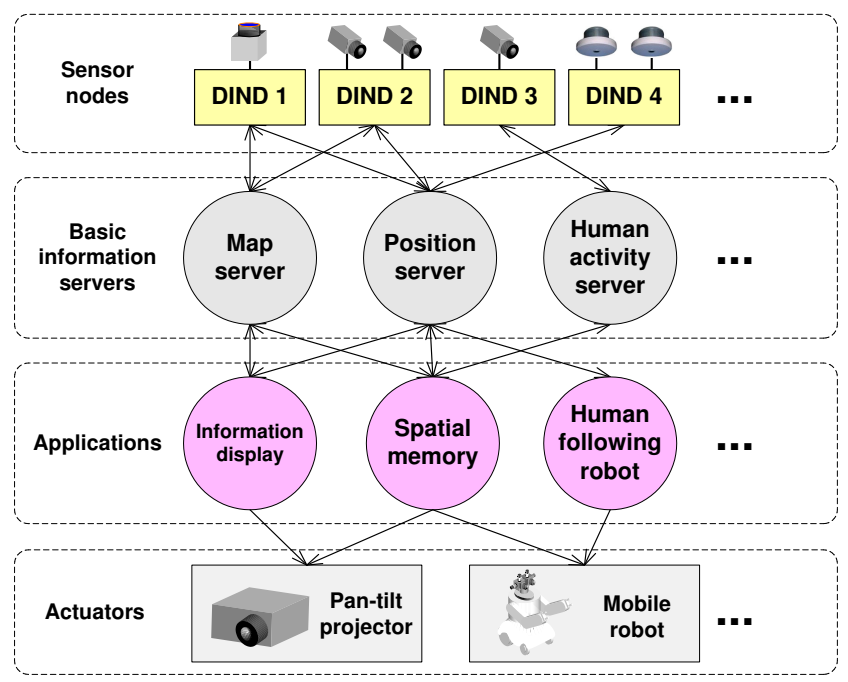

Fig. 3. Configuration of a sensor cluster in Intelligent Space

the top of the mobile robot. The mobile robot is also equipped with a wireless network device to communicate with iSpace.

Fig. 3 shows the configuration of the sensor cluster in iSpace, at which information from all sensor nodes are fused and the combined information is utilized by the applications to perform informative and physical actuation. It consists of four layers - the sensor node layer, the basic information server layer, the application layer and the actuator layer.

The information obtained by each sensor node is first sent to the corresponding basic information servers with the reliability of the measurement, which is used for information fusion. For example, geometrical shape of the environment and position of the target are sent to the map server and the position server respectively. That is, each sensor node sends the information that can be obtained. The basic information server combines the information based on the reliability and, if necessary, sends the result back to the sensor nodes. The returned information can be used for the next measurement in the sensor node. 
The applications request the information they need from basic information servers. Based on the received information, the applications send the commands to the actuators and realize various services to the users. For example, an information display system would get the map of the space and the position of the humans from the map and position server respectively, find an empty area in front of the human and project an image using a projector. The applications only need to know the basic information server's address to get information, so they don't need to connect directly to the sensor which observes the target object. In addition, with this structure it is possible to add, remove or replace the sensor devices or sensor nodes without any change in the application program.

\section{AUTOMATED CALIBRATION OF THE DISTRIBUTED LASER RANGE FINDERS}

\subsection{Overview of Proposed Method}

In this section, the problem of the calibration of the distributed sensors is addressed. As mentioned in section 1, we utilize the moving objects in iSpace to realize the automated calibration. Here we consider the automated calibration of the pose of the laser range finders. Laser range finders have recently been frequently used for a variety of sensing tasks, most notably as on-board sensor for mobile robots. This is probably due to the appearance of relatively low priced eye-safe laser range finder devices. They also have some advantages for intelligent environment applications. For example, their installation is simple and tracking targets don't need to have special tags. Moreover, they can be used for both object tracking and map building.

Fig. 4 shows the overview of the calibration method. Let ${ }^{\mathrm{L} 1} \mathrm{O}-{ }^{\mathrm{L} 1} \mathrm{x}^{\mathrm{L} 1} \mathrm{y}$ be the coordinate system fixed to the laser range finder 1 and ${ }^{\mathrm{L} 2} \mathrm{O}-{ }^{\mathrm{L} 2} \mathrm{x}^{\mathrm{L} 2} \mathrm{y}$ be the coordinate system fixed to the laser range finder 2. First, each laser range finder tracks moving objects and gets their positions in its local coordinate system. If these sensors have overlapping observation regions, position of the same object at time $k$ in each laser range finder's coordinate system $\left(x_{k 1}, y_{k 1}\right),\left(x_{k 2}, y_{k 2}\right)$ is acquired as corresponding point. The calibration process is then performed based on the set of corresponding points $\left\{\left(x_{k 1}, y_{k 1}\right),\left(x_{k 2}, y_{k 2}\right)\right\}$ $(k=1,2, \ldots, n)$. The calibration parameters are relative position and orientation of two laser range finders in $2 \mathrm{D}$ plane $\left(T_{x}, T_{y}, \theta\right)$.

In the following subsections, the functions needed for the proposed calibration method are described.

\subsection{Tracking Process}

The tracking process consists of background subtraction, clustering, data association and tracking using the Kalman filter, which are performed on each DIND with the laser range finder. Each of these steps is described in the following.

Background subtraction Background subtraction is processes of determining which parts of the scan are due to static objects, and which come from reflections from

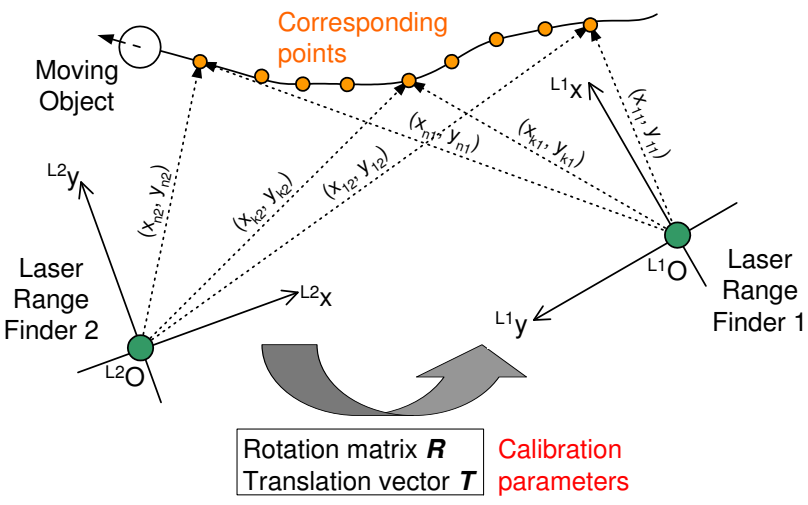

Fig. 4. Overview of the calibration method

moving objects, which in the case of iSpace are mainly humans and robots. The part belonging to static objects is called the background, whereas the part belonging to moving objects is called the foreground. The background can be easily determined by taking several scans while there are no moving objects in the space and taking their average.

The foreground (moving objects) can be extracted from the scan by comparing with the learned background: the parts of the scan that differ from the background more than a given threshold are marked as foreground.

Clustering and data association The scan points in the foreground are clustered based on the Euclidian distance between them using a nearest neighbor classifier. This divides the foreground to a number of clusters, each belonging to one of the tracked object. Clusters with a small number of scan points ( 1 or 2 points) are discarded, which effectively eliminates possible noise in the measurements.

In order to determine which cluster belongs to which tracked object the cluster centers, which are obtained by averaging the position of all points in the cluster, are compared with the positions of currently tracked objects, and each cluster is assigned to the closest object. The clusters that are far from all currently tracked objects are considered as new objects, and a new tracking process is started for them. This data association based on the Euclidian distance is very simple, but it proved to be enough in our experiments where there were not many tracked objects in the space.

Tracking using the Kalman filter From the previous step the positions of cluster centers were obtained. But since the objects are scanned from one side, the center of the obtained cluster of points in general does not coincide with the center of the tracked object. This may lead to a large calibration error in case that the object is scanned from two different sides by two laser range finders. Therefore, at first, we need to estimate object center from the cluster center. Here we assume that the shape of the object is almost circular. To obtain the positions of the tracked object from the cluster positions we use an approximate relation described by the following equations (Fig. 5):

$$
\begin{aligned}
& x_{o b j}=x_{c l}+d \cos \alpha \\
& y_{o b j}=y_{c l}+d \sin \alpha
\end{aligned}
$$




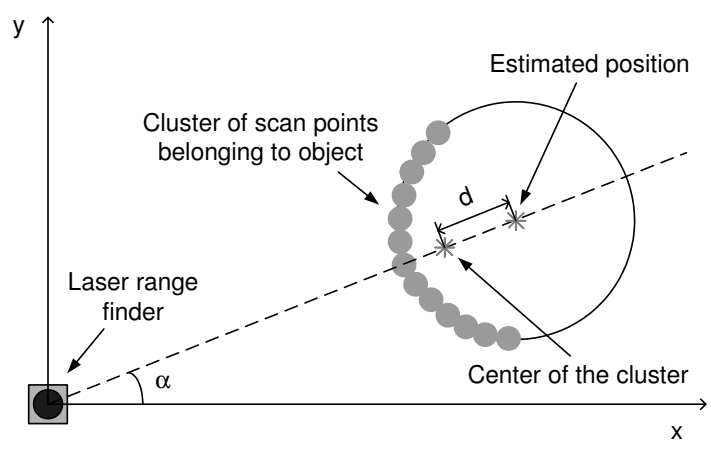

Fig. 5. Estimation of object center

where $x_{c l}$ and $y_{c l}$ are the cluster center coordinates, $\alpha$ is the angle of the line between the laser range finder and the center of the cluster, and $d$ is a parameter depending on the radius of the object. In our experiments $d$ was set to 6 $\mathrm{cm}$ for human (i.e. human's leg) and $15 \mathrm{~cm}$ for the mobile robot.

In the case of tracking robots, the result of (1) can be considered as a measurement of the robot position. But in the case of tracking a human, the position has to be determined based on two clusters belonging to his/her legs, as noted earlier. In a given measurement step these clusters may or may not be available depending on occlusions in the scan. Since the position of the human can be assumed to be in the middle between both legs, in case both legs are visible the measurement of the human's position is taken as their mean value. When only one leg is visible the other leg's position is assumed based on the current human's tracked position, and the measured position is calculated accordingly, but this time the measurement is considered less reliable.

The number of clusters belonging to an object can be used as a simple way to distinguish between humans and other objects. We use it to determine which of the described ways to use to calculate the object position. By taking the previously described position measurements a Kalman filter can be applied to track the objects. Since we assume no specific knowledge of the object motion model, the tracked objects (both human and robot) are described using the following linear state space model:

$$
\begin{aligned}
& \boldsymbol{x}_{k}=\boldsymbol{A} \boldsymbol{x}_{k-1}+\boldsymbol{w}_{k-1} \\
& \boldsymbol{z}_{k}=\boldsymbol{H} \boldsymbol{x}_{k-1}+\boldsymbol{v}_{k}
\end{aligned}
$$

where the state consists of the $(x, y)$ position and speeds in the $x$ and $y$ direction, and the outputs are the coordinates of the corresponding cluster center. The process and measurement noises described with matrices $\boldsymbol{w}$ and $\boldsymbol{v}$ are assumed to be Gaussian with zero mean. The matrices $\boldsymbol{A}$ and $\boldsymbol{H}$ in (2) have the following form:

$$
\boldsymbol{A}=\left[\begin{array}{cccc}
1 & 0 & T_{d} & 0 \\
0 & 1 & 0 & T_{d} \\
0 & 0 & 1 & 0 \\
0 & 0 & 0 & 1
\end{array}\right], \quad \boldsymbol{H}=\left[\begin{array}{cccc}
1 & 0 & 0 & 0 \\
0 & 1 & 0 & 0
\end{array}\right]
$$

where $T_{d}$ is the sample time. Based on the described model the Kalman filter equations Thrun et al. [2005] are then applied recursively to obtain an estimate of the position and speed of the tracked object.

\subsection{Calibration Process}

In the calibration process, the relative position and orientation of two laser range finders $\left(T_{x}, T_{y}, \theta\right)$ is calculated from the set of corresponding points $\left\{\left(x_{k 1}, y_{k 1}\right),\left(x_{k 2}, y_{k 2}\right)\right\}$ $(k=1,2, \ldots, n)$. We solve the least square error problem denoted by the following equation:

$$
\begin{array}{r}
\epsilon^{2}=\sum_{i=1}^{n}\left\{\left(x_{i 1}-\cos \theta x_{i 2}+\sin \theta y_{i 2}-T_{x}\right)^{2}\right. \\
\left.+\left(y_{i 1}-\sin \theta x_{i 2}-\cos \theta y_{i 2}-T_{y}\right)^{2}\right\}
\end{array}
$$

where the indices 1 and 2 represent the obtained laser range finder 1 and 2 coordinates. If more than one set of corresponding points are obtained, we can derive the following estimates from $\frac{\partial \epsilon^{2}}{\partial T_{x}}=0, \frac{\partial \epsilon^{2}}{\partial T_{y}}=0$ and $\frac{\partial \epsilon^{2}}{\partial \theta}=0$ :

$$
\begin{aligned}
& T_{x}=\mu_{x 1}-\cos \theta \mu_{x 2}+\sin \theta \mu_{y 2} \\
& T_{y}=\mu_{y 1}-\sin \theta \mu_{x 2}-\cos \theta \mu_{y 2}
\end{aligned}
$$

$$
\begin{array}{r}
\theta= \\
\operatorname{atan} 2\left\{\sum_{i=1}^{n} \frac{-\left(x_{i 1} y_{i 2}-y_{i 1} x_{i 2}\right)}{n}+\mu_{x 1} \mu_{y 2}-\mu_{y 1} \mu_{x 2},\right. \\
\left.\sum_{i=1}^{n} \frac{\left(x_{i 1} x_{i 2}+y_{i 1} y_{i 2}\right)}{n}-\mu_{x 1} \mu_{x 2}-\mu_{y 1} \mu_{y 2}\right\}
\end{array}
$$

where $\mu$ 's stand for mean values, for example:

$$
\mu_{x 1}=\frac{1}{n} \sum_{i=1}^{n} x_{i 1}
$$

\subsection{Finding corresponding object among DINDs}

In case that mobile robots are used as calibration objects, the model of the mobile robots can be used to determine which mobile robots are being tracked. However, if the general moving object is utilized, we have to judge whether two tracked objects in different sensors are same object or not.

When the estimation is done based on the correct corresponding paths, the average of square error for each point $\epsilon / n$ which is derived from (4) is expected to have a small value. So, calibration parameters are calculated for each combination of the currently tracked object in one DIND and objects tracked in other DINDs at the same time and, if $\epsilon / n$ is lower than a certain threshold, the paths are considered to be same object's. The validity of this method is discussed in the next section.

\section{EXPERIMENT}

We made an experiment in order to evaluate the method for finding same object.

Two laser range finders LRF1 and LRF2 are placed in the environment such that the observation areas have overlapping. Here the coordinates of LRF1 is used as a reference coordinate system and the transformation parameters from the LRF2 coordinates to the LRF1 coordinates 


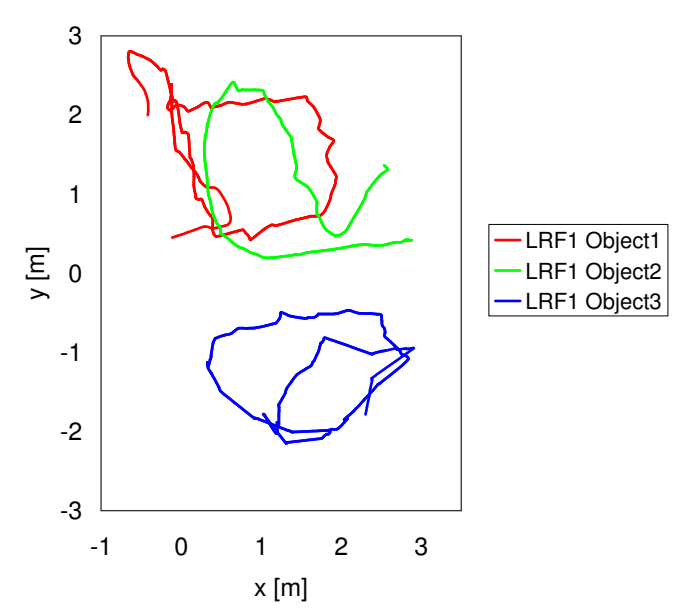

Fig. 6. Tracked positions of people in LRF1

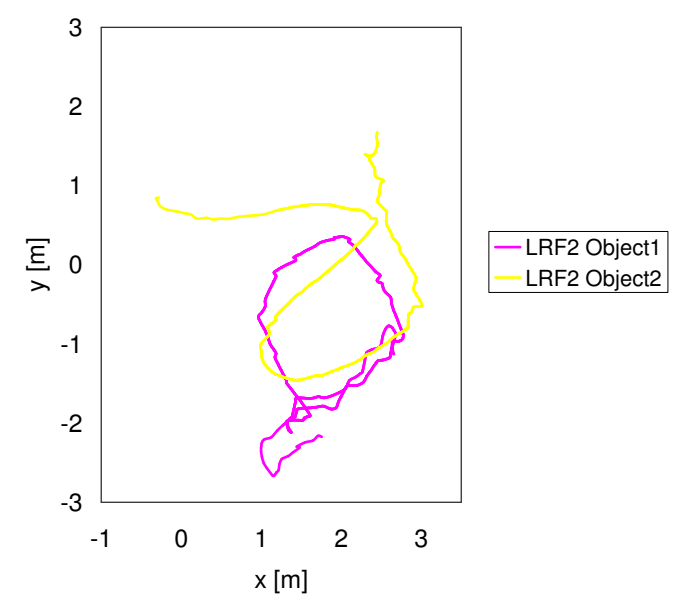

Fig. 7. Tracked positions of people in LRF2

are calculated. By manual calibration, the transformation parameters are found as $T_{x}=2.22 \mathrm{~m}, T_{y}=2.83 \mathrm{~m}$, $\theta=1.96 \mathrm{rad}$. In the case of manual calibration, a calibration object (an object which can be well detected by a laser range finder) is placed in turn on several points with known global coordinates and the calibration parameters are calculated by $(5)-(7)$.

In this experiment, three people walked inside the environment simultaneously. The results of human tracking in the LRF1 and the LRF2 are shown in Fig. 6 and Fig. 7, respectively. The LRF1 tracked all three people whereas the LRF2 could track only two people since a person didn't move in the overlapping observation region.

Tab. 1 shows the result of the automated calibration and the mean of the square error for all combinations of the obtained paths. For the correct corresponding paths (i.e. Object1 in LRF1 and Object1 in LRF2, Object2 in LRF1 and Object2 in LRF2), the average of the estimation error for each point is small and the estimated parameters are almost same as the result of the manual calibration. This means that the corresponding paths can be detected based on the estimation error even if multiple people exist in the environment. However, when two people happen to make similar movement at the same time, the estimation error gets small despite the wrong correspondence. So, a
Table 1 . Result of the automated calibration

\begin{tabular}{|c|c|c|c|c|c|}
\hline \multicolumn{2}{|c|}{ Object ID } & \multirow{2}{*}{$\begin{array}{l}T_{x} \\
{[\mathrm{~m}]}\end{array}$} & \multirow{2}{*}{$\begin{array}{l}T_{y} \\
{[\mathrm{~m}]}\end{array}$} & \multirow{2}{*}{$\begin{array}{c}\theta \\
{[\mathrm{rad}]}\end{array}$} & \multirow{2}{*}{$\begin{array}{l}\epsilon^{2} / n \\
{\left[\mathrm{~m}^{2}\right]}\end{array}$} \\
\hline LRF1 & LRF2 & & & & \\
\hline 1 & 1 & 2.22 & 2.83 & -1.93 & $1.34 \times 10^{-2}$ \\
\hline 1 & 2 & 0.20 & -0.15 & 1.38 & 1.48 \\
\hline 2 & 1 & -0.61 & 0.80 & 0.61 & $4.19 \times 10^{-1}$ \\
\hline 2 & 2 & 2.14 & 2.95 & -1.87 & $5.83 \times 10^{-3}$ \\
\hline 3 & 1 & -0.23 & -0.25 & 0.00 & 1.30 \\
\hline 3 & 2 & 3.59 & -1.61 & 2.99 & 1.57 \\
\hline
\end{tabular}

voting strategy which selects a frequently appeared value as the estimate, is one solution for robust estimation since the computed calibration parameters are approximately constant for correct correspondences. For example, in Tab. 1 row 1 and 4 have both similar estimates and small errors, so they represent the correct estimates.

\section{CONCLUSION}

In this paper, we presented the automated calibration of the distributed laser range finders by using the overlapping sensing regions and the result of moving object tracking. The positions of the moving objects in each sensor's coordinate system are stored as corresponding points and the calibration process is then performed based on the set of corresponding points. If a general moving object is utilized, we have to judge whether two tracked objects in different sensors are same object or not. So the paths which lead to small estimation error are considered as same object's. The experimental result shows that this method can find correct correspondence and achieve almost the same result as manual calibration case.

For future work, we will evaluate the proposed method in a larger area with more laser range finders. In addition, since the proposed calibration method is affected by the error of the object tracking, especially estimation of the object center, we consider utilization of background information for accuracy improvement. This background information is also useful for finding the same object in two different sensors.

\section{REFERENCES}

D. J. Cook and S. K. Das. Smart Environments: Technologies, Protocols, and Applications (Wiley Series on Parallel and Distributed Computing). Wiley-Interscience, 2004.

A. Galstyan, B. Krishnamachari, K. Lerman and S. Pattem. Distributed Online Localization in Sensor Networks Using a Moving Target. Proc. of Third Int. Symposium on Information Processing in Sensor Networks, pages 61-70, 2004.

A. Howard, M.J. Mataric and G. Sukhatme. Relaxation on a Mesh: a Formalism for Generalized Localization. Proc. of IEEE/RSJ Int. Conf. on Intelligent Robots and Systems, volume 2, pages 1055-1060, 2001.

B. Johanson, A. Fox and T. Winograd. The Interactive Workspaces Project: Experiences with Ubiquitous Computing Rooms. IEEE Pervasive Computing, volume 1, number 2, pages 67-74, 2002.

Y. Koide, T. Kanda, Y. Sumi, K. Kogure and H. Ishiguro. An Approach to Integrating an Interactive Guide Robot with Ubiquitous Sensors. Proc. of the 2004 IEEE/RSJ 
Int. Conf. on Intelligent Robots and Systems, pages 2500-2505, 2004.

J.-H. Lee and H. Hashimoto. Intelligent Space - Concept and Contents, Advanced Robotics, volume 16, number 3, pages 265-280, 2002.

F. Mizoguchi, H. Ohwada, H. Nishiyama and H. Hiraishi. Smart Office Robot Collaboration Based on MultiAgent Programming. Artificial Intelligence, volume 114, issue 1-2, pages 57-94, 1999.

T. Mori, N. Hayama, H. Noguchi and T. Sato. Informational Support in Distributed Sensor Environment Sensing Room. Proc. of 13th IEEE Int. Workshop on Robot and Human Interactive Communication, pages 353-358, 2004.

E. D. Mynatt, A.-S. Melenhorst, A.-D. Fisk and W. A. Rogers. Aware Technologies for Aging in Place: Understanding User Needs and Attitudes. IEEE Pervasive Computing, volume 3, number 2, pages 36-41, 2004.

Y. Nishida, T. Hori, T. Suehiro and S. Hirai. Sensorized Environment for Self-Communication Based on Observation of Daily Human Behavior. Proc. of IEEE/RSJ Int. Conf. on Intelligent Robots and Systems, volume 2, pages 1364-1372, 2000.

I. Rekleitis and G. Dudek. Automated Calibration of a Camera Sensor Network. Proc. of IEEE/RSJ Int. Conf. on Intelligent Robots and Systems, pages 3384-3389, 2005.

T. Sasaki, D. Brscic and H. Hashimoto. Implementation of Distributed Sensor Network for Intelligent Space. Proc. of the IEEE Int. Conf. on Mechatronics, TuA1-B-1, 2007.

A. Sgorbissa and R. Zaccaria. The Artificial Ecosystem: A Distributed Approach to Service Robotics. Proc. of the 2004 IEEE Int. Conf. on Robotics and Automation, volume 4, pages 3531-3536, 2004.

S. Shenoy and J. Tan. Simultaneous Localization and Mobile Robot Navigation in a Hybrid Sensor Network. Proc. of IEEE/RSJ Int. Conf. on Intelligent Robots and Systems, pages 1636-1641, 2005.

S. Thrun, W. Burgard and D. Fox. Probabilistic Robotics. MIT Press, 2005. 